Europhys. Lett., 58 (5), pp. 693-698 (2002)

\title{
Prandtl and Rayleigh numbers dependences in Rayleigh-Bénard convection
}

\author{
P.-E. Roche ${ }^{1,2}$, B. Castaing ${ }^{1,3}$, B. Chabaud ${ }^{1}$ and B. Hébral ${ }^{1}$ \\ 1 Centre de Recherches sur les Très Basses Températures \\ associé à l'Université Joseph Fourier - 38042 Grenoble Cedex 9, France \\ 2 Laboratoire de Physique de la Matière Condensée, Ecole Normale Supérieure \\ 24 rue Lhomond, 75231 Paris Cedex 5, France \\ 3 Ecole Normale Supérieure de Lyon - 46 allée d'Italie, 69364 Lyon Cedex 7, France
}

(received 27 September 2001; accepted in final form 14 March 2002)

PACS. 47.27.-i - Turbulent flows, convection, and heat transfer.

PACS. 44.25.+f - Natural convection.

PACS. 67.90.+z - Other topics in quantum fluids and solids; liquid and solid helium (restricted to new topics in section 67$)$.

\begin{abstract}
Using low-temperature gaseous helium close to the critical point, we investigate the Prandtl-number dependence of the effective heat conductivity (Nusselt number) for a $1 / 2$ aspect ratio Rayleigh-Bénard cell. Very weak dependence is observed in the range $0.7<$ $\operatorname{Pr}<21 ; 2 \times 10^{8}<R a<2 \times 10^{10}$ : the absolute value of the average logarithmic slope $\delta=(\partial \ln N u / \partial \ln P r)_{R a}$ is smaller than 0.03. A bimodality of $N u$, with $7 \%$ difference between the two sets of data, is observed, which could explain some discrepancies between precise previous experiments in this range.
\end{abstract}

One century of experimental and theoretical studies have not succeeded in understanding the mechanism of heat transfer in turbulent convection. Since the work of Lord Rayleigh [1], in 1916, a basic geometry has focused most physicists' attention: the Rayleigh-Bénard cell consists in a layer of fluid enclosed between two isothermal horizontal plates. A temperature difference $\Delta T$ between the plates forces the fluid convection. In comparison with the diffusive heat transport, convection enhances heat transfer by a factor called the Nusselt number $(\mathrm{Nu})$. The Boussinesq approximation states that the fluid properties are temperature independent. In this case, let apart the cell aspect ratio, $N u$ only depends on two parameters: the dimensionless $\Delta T$, called the Rayleigh number $(R a)$, and the ratio of the kinematic $(\nu)$ and thermal $(\kappa)$ diffusivities: the Prandtl number $(\operatorname{Pr}=\nu / \kappa)$ [2].

In most of the studies, the experimental $N u(R a)$-dependence has been compared to theoretical predictions. But the limited range of $R a$ explored (rarely more than 1.5 decade) and experimental complications made the discrimination between theories difficult [3]. For instance, recent precise measurements [4] seemed to rule out a single power law behavior on any significant range. But it was later realized $[5,6]$ that considering side wall effects raises some doubts on this conclusion. 


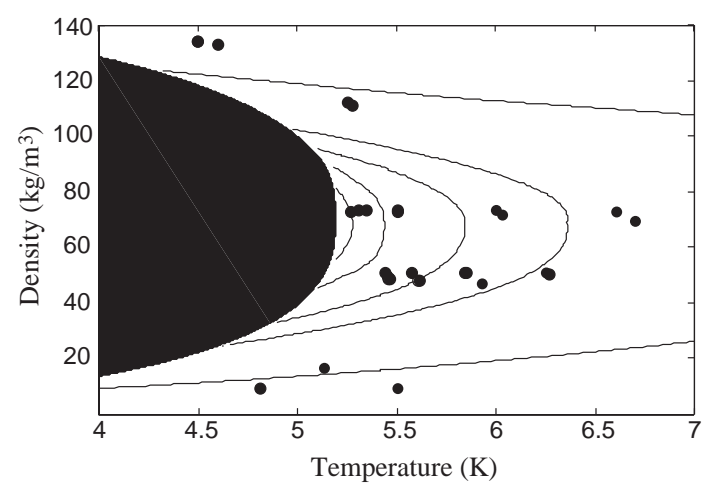

Fig. 1

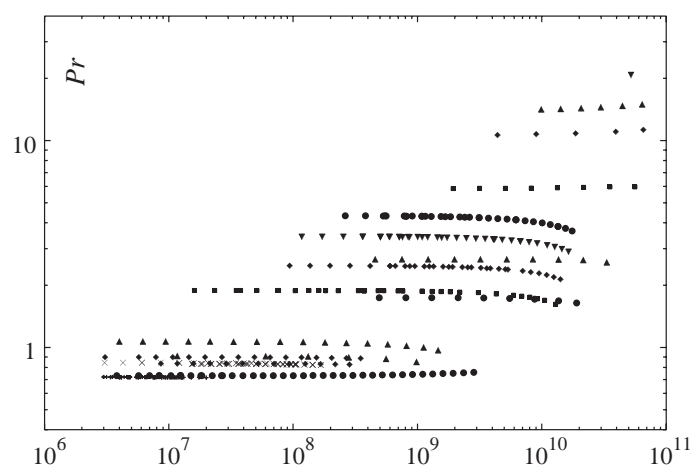

Fig. 2

Fig. 1 - Prandtl-number contours $\operatorname{Pr}=1,2,3,7,21$ in the density-temperature plane. The highest $\operatorname{Pr}$ are obtained close to the critical point. The dots pin explored $T, d$ parameters.

Fig. 2 - Explored $R a$ and $\operatorname{Pr}$ parameters space.

Recently, Grossmann and Lohse [7] proposed a theory which has the advantage to lay on simple accepted ideas. Thus, it is important to know if it can fit the data or if these ideas fail in some way. The $N u(P r)$-dependence put more constraints on this theory and the previous ones than $N u(R a)$, especially in the middle $\operatorname{Pr}$ range $(P r \simeq 1)$, where one shifts from low- $P r$ to high- $\operatorname{Pr}$ regimes.

But experimental variation of $P r$ is not straightforward and requires a specially designed experiment. By varying the temperature of water, Liu and Ecke obtained a $\operatorname{Pr}$ variation over 1/4 decade (3.75-6.75) [8] while in ref. [9] Ahlers and Xu employed various fluids to obtain 4 different $\operatorname{Pr}$ numbers, over a 1 decade range (4-34). The results of these two groups are in contradiction with those obtained by Ashkenazi and Steinberg in the critical region of $S F_{6}$ over two decades of $\operatorname{Pr}(1-93)$ [10]. For completeness, it is worth mentioning the numerical results of Verzicco and Camussi [11] and those of Kerr and Herring [12]. After completion of this work, we had also knowledge of recent measurements by Xia et al. [13], at high $\mathrm{Pr}$.

In this paper we report turbulent heat transport measurements over nearly 4.5 decades of $R a$ and 1.5 decades of $P r$. The variation of $P r$ is achieved by setting the average temperature and density of helium in the vicinity of its critical point $\left(T \simeq 5.19 \mathrm{~K}\right.$ and $\left.d \simeq 69.6 \mathrm{~kg} \mathrm{~m}^{-3}\right)$. This procedure, already employed in $S F_{6}$ [10], is motivated by the divergence of $\operatorname{Pr}$ at the critical point, although we limit ourselves to the far tail of this $\operatorname{Pr}$ peak. Figure 1 shows contour plots of $\operatorname{Pr}(T, d)$ and present data. Figure 2 shows their distribution in the $(\operatorname{Ra}, \operatorname{Pr})$ plane.

Cryogenic helium was chosen as the convection fluid for various experimental reasons appearing in the set-up description. They include: negligible heat leaks corrections, very low electrical noise environment, and favorable properties of helium, allowing large values of the coefficient $k: R a=k \alpha \Delta T ; k=g h^{3} /(\nu \kappa)$, where $h$ is the height of the cell, $g$ the gravity constant, and $\alpha$ the constant-pressure thermal-expansion coefficient. The low helium critical pressure $(2.27$ bar $)$ allows the use of a thin side wall $(250 \mu \mathrm{m})$. The size and shape of the cell are chosen to allow direct comparison with previous works. The shape is the same as in $[4,9-11,14-17]$. The height $(2 \mathrm{~cm})$ is such that the maximum $R a$ obtained, close to the critical point at large $P r$, are smaller than the transition towards the Kraichnan regime $[3,14,17]$. 


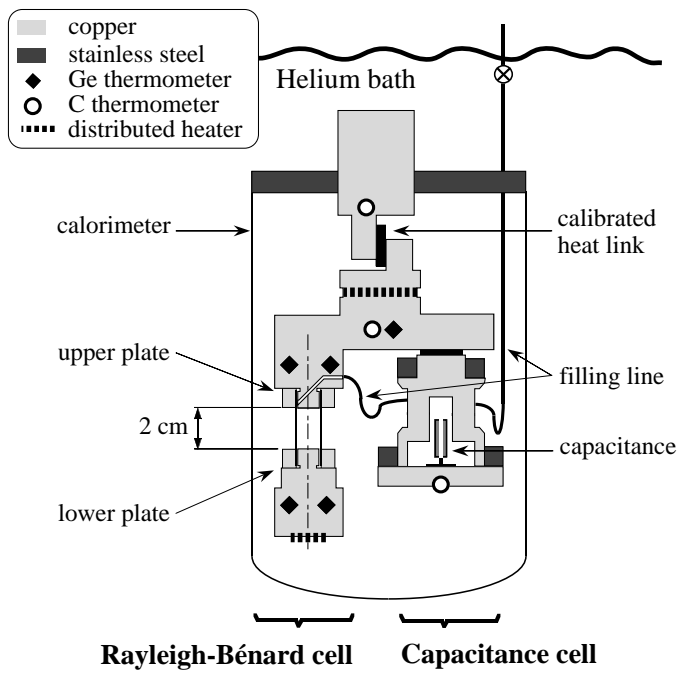

Fig. 3 - Experimental set-up. Convection in the capillary tubes is prevented by two thermal siphons.

Figure 3 is a schematic view of the set-up. The cylindrical convection cell, $2 \mathrm{~cm}$ high and $1 \mathrm{~cm}$ in diameter (0.5 aspect ratio), is hanging in a cryogenic vacuum. The stainless-steel wall conductance is measured as a function of temperature $(147 \mu \mathrm{W} / \mathrm{K}$ at $5 \mathrm{~K})$. Note that the wall thickness is constant along the whole cell height. This allows to correct data for the wall conductance according to [6]. Heat leaks from the bottom plate to the calorimeter are negligible in such a set-up, as shown in [18]. Direct measurements, conducted with a cryogenic vacuum in the cell (first run of the cell), confirmed this point.

The bottom and top plates are made of copper. The latter one is in thermal contact with the liquid-helium bath through a measured $62 \mathrm{~K} / \mathrm{W}$ heat link (at $5 \mathrm{~K}$ ) and its temperature is regulated by a PID controler with a stability better than $10 \mu \mathrm{K}$. The bottom plate is Joule heated with a constant power. A constant strain gauge is used as a distributed heater.

Temperatures are deduced from the resistance of germanium thermometers. Their thermalcycling calibrations offsets are adjusted, in situ, to better than $0.4 \mathrm{mK}$ with respect to the critical temperature. The resistance measurements are performed through 4 low-thermalconduction superconducting wires. We have 4 germanium thermometers, two in each plate. We can directly measure the ratio between two of these resistances (one in each plate) through a ratio bridge [19], whose zeroing is performed systematically, before heating the bottom plate. The change of this ratio when heating gives access to the temperature difference $\Delta T$. The correction corresponding to the finite conductivity of the plates is always very small. This procedure makes possible measurements of the adiabatic gradient effect for $\Delta T \sim \Delta T_{\text {adia }}$, where $\Delta T_{\text {adia }} \sim 40-80 \mu \mathrm{K}$ is the temperature difference associated with the adiabatic gradient. Present data are restricted to $\Delta T \geq 5 \Delta T_{\text {adia }}$ and to Boussinesq criterion $\alpha \Delta T \leq 20 \%$. $R a$ and $N u$ are corrected for the adiabatic-gradient effect according to the exact formula presented in [2] and [14].

The helium density is measured in situ, in a copper cell in thermal contact with the top plate, from a dielectric-constant measurement, made through an immersed capacitance. In situ calibration is performed on the whole density range with a $0.1 \%$ resolution. At the beginning of each run, before heating the bottom plate, the convection cell has the same pressure and temperature, and thus the same density, as the copper cell. Then, during the 


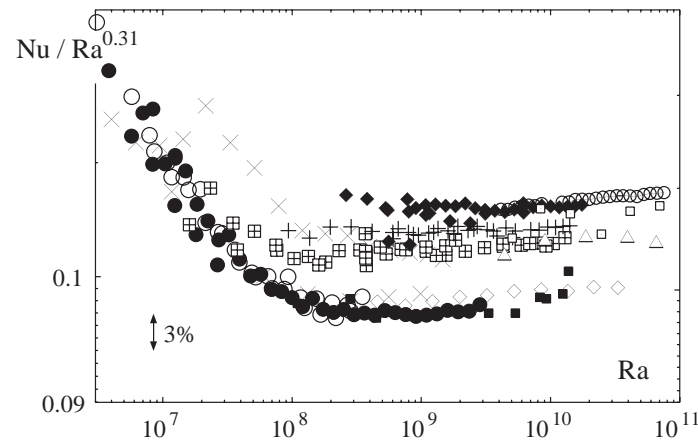

Fig. 4



Fig. 5

Fig. $4-\bullet P r \simeq 0.73, \circ P r \simeq 0.87, \times P r \simeq 0.95, \boxplus P r \simeq 1.6-1.9,+P r \simeq 2.1-2.5, \diamond P r \simeq 2.6$, $\checkmark P r \simeq 3.6-4.3, \triangle P r \simeq 10.6-11.3$. For comparison, data obtained in cells of the same aspect ratio are plotted with smaller symbols: the circled continuous line is from ref. [9] $(\operatorname{Pr}=3.97), \square(\operatorname{Pr}=1.1)$ and $\mathbf{~}(\operatorname{Pr}=0.7)$ are from ref. [17]. It should be noted that data from ref. [9] cannot be corrected for side wall conduction with the same procedure as used for others data because of the thickness variation of the side wall. The correction proposed by the authors [5] is indeed employed.

Fig. $5-N u$ vs. $\operatorname{Pr}$ for different $R a$. Filled symbols: this work. $\nabla R a=2 \times 10^{7}, \Delta R a=2 \times 10^{8}$, - $R a=2 \times 10^{9}$, $R a=1.4 \times 10^{10}, \quad R a=5.23 \times 10^{10}$. Open symbols: data from ref. [9]. $\circ$ $R a=2 \times 10^{9}, \square R a=1.4 \times 10^{10}, \diamond R a=5.23 \times 10^{10}$. The continuous line represents the $\mathrm{Pr}^{-1 / 7}$ power law dependence predicted by the $2 / 7$ law models [3].

run, the total mass (copper cell + convection cell) is kept constant. As the density in the copper cell can be measured at each time, the density in the convection cell is always known. The helium properties are deduced from this density and the average temperature, based on a compilation from various sources [20]. Improvement over published fits concerns transport properties and the connection between the fits of Kierstead and McCarty. This improvement is supported by in situ measurement of the thermal-expansion coefficient: the total mass in both cells being fixed, the measured density changes when the average temperature of the convection cell differs from the copper one.

Below $R a=10^{6}$, the onset-of-convection region is used as a test regime for the thermal resolution of the set-up: in this region, the cell is indeed operated at a very low level of heating (a few hundred nanowatts, to be compared to a typical heating of $250 \mu \mathrm{W}$ ). The onset $R a$ number found is $4 \times 10^{4}$. The strong difference with the infinite aspect ratio value (1709) is due to the 0.5 aspect ratio and is in agreement with previous measurements in cells of low side wall conductivity $[17,18]$.

In fig. 4, we show $N u$ as a function of $R a$. In order to display all data with an increased vertical resolution, $N u$ is renormalized by $R a^{0.31}$. Note that the 0.31 exponent is only a "best-fit" value and we do not suggest that $N u$ should follow a power law. For comparison, we display the data from ref. [17] $(P r \simeq 0.7)$ and $[9](P r \simeq 4)$, which were obtained with the same cell geometry.

For $2 \times 10^{7}<R a<2 \times 10^{10}$, points can be separated into two subsets which differ by roughly $7 \%$ in $N u$ (fig. 4). Such a data bimodality cannot be taken into account by the uncertainties, which are twice smaller than this $N u$ gap. In addition, switches from one set of data to the other occur within series of points of same average temperature and density conditions. For such series of points, the relative resolution on $N u$ is much better than the 
absolute resolution mentioned above, since the thermodynamical and transport properties are kept constant. Such switches can also be seen in the data from ref. [17].

We cannot decide if this bistability reveals spurious effect of boundary conditions (walls finite specific heat, for instance), is of hysteretic nature, or reveals the intrinsically random nature of $\Delta T$ averaged on large (hundreds of turn-over times) but finite times. The above observation of shifts would favor the last hypothesis. Let us make two remarks:

i) The huge correlation time it implies (several thousands of turn-over times) for $\Delta T$ prevents one from performing a real ergodic average of $N u$ in a single run. Due to its small size, our cell has the lowest turn-over time in this $R a$ range.

ii) Such an average $N u$ would correspond to a $\Delta T$ value which is never observed, in the middle of the two data sets.

It is interesting also to note the apparent invariance of the $N u$ gap (on a log scale), which suggests a single mechanism for local heat transfer. Such double-valued $N u$ have already been reported in the low Rayleigh numbers region, where they are associated to the different largescale circulation modes [21]. This modal interpretation remains appealing in our $R a$ range, although it alters the dogma of the stability of turbulent flow in typical Rayleigh-Bénard cells. It could explain some of the discrepancies between published results $[9,15,16,22]$.

We finally want to address the $P r$ - and $R a$-dependences of $N u$. In fig. 5, data are presented as a function of $\operatorname{Pr}$ for 4 differents $R a$ numbers. In this plot, the symbols size is slightly larger than our vertical error bars. For comparison, the data from ref. [9] are also plotted. On this scale, the bimodality mentioned above is only slightly visible. As can be seen, our data are in contradiction with the $\mathrm{Pr}^{-1 / 7}$-dependence reported in [10]. Note also that the multivalued character of $N u$ does not affect the local exponent $\gamma=\partial \log (N u) / \partial \log (R a)$.

From fig. 4, two $R a$ ranges can be distinguished by a sharp increase of the local exponent $\gamma$. The threshold $R a$ number is $1-2 \times 10^{8}$, which well corresponds to the soft-to-hard turbulent transition [23] in 0.5 aspect ratio cylindrical cell [16]. Above $R a=2 \times 10^{8}$, if we fit the whole data set by a single average power law of the type $N u \sim P r^{\delta} \cdot R a^{\gamma}$, we obtain $0.31<\gamma<0.32$ and $|\delta|<0.03$, for $0.7<\operatorname{Pr}<11$. It is interesting to note that this statement can be extended to $\operatorname{Pr}=14.2$ and $\operatorname{Pr}=34.1$, according to Ahlers and Xu data [9]. This is hardly compatible with the last decade most studied theories of turbulent convection, which predict $N u \sim \operatorname{Pr}^{-1 / 7} \cdot \operatorname{Ra}^{2 / 7}[3]$.

A totally different analysis of the data can be made in the perspective of the recent model of Grossman and Lohse [7]. In this model, the effective exponents $\delta$ and $\gamma$ are a function of $R a, P r$ and of 5 aspect-ratio-dependent parameters. Complete and precise determination of the limits our data impose to these parameters is a delicate piece of work for which we refer to these authors. Let us note, anyway, that the independence of $N u$ from $\operatorname{Pr}$ down to $\operatorname{Pr}=0.7$ is a severe constraint on this theory which aims to describe also the low- $P r$ regime, where a strong dependence is observed [24].

In conclusion, the main result of this paper is the independence of $N u$ from $\operatorname{Pr}$ for $2 \times 10^{8}<$ $R a<2 \times 10^{10}$ and $0.7<\operatorname{Pr}<21$. One can even push the upper limit on $\operatorname{Pr}$ up to 34 if we use our agreement with Ahlers and $\mathrm{Xu}$ data [9]. The bimodality we observe, probably due to the large-scale motion, reconciliates the published data with this geometry and within this range if we except the $S F_{6}$ experiment [10].

Acknowledgements are due to J. Niemela, D. Lohse and R Verzicco for fruitful discussions, and V. ARP for help in retrieving the literature on helium properties. 


\section{REFERENCES}

[1] Lord Rayleigh, Philos. Mag., 32 (1916) 529.

[2] Tritton D. J., Physical Fluid Dynamics (Oxford University Press) 1988.

[3] Siggia E., Annu. Rev. Fluid Mech., 26 (1994) 137.

[4] Xu X., Bajaj K. M. S. and Ahlers G., Phys. Rev. Lett., 84 (2000) 4357.

[5] Ahlers G., Phys. Rev. E, 63 (2001) 015303(R).

[6] Roche P.-E., Castaing B., Chabaud B., Hébral B. and J. Sommeria, Eur. Phys. J. B, 24 (2002) 405.

[7] Grossmann S. and Lohse D., Phys. Rev. Lett., 86 (2001) 3316.

[8] Liu Y. and Ecke R. E., Phys. Rev. Lett., 79 (1997) 2257.

[9] Ahlers G. and Xu X., Phys. Rev. Lett., 86 (2001) 3320.

[10] Ashrenazi S. and Steinberg V., Phys. Rev. Lett., 83 (1999) 3641.

[11] Verzicco R. and Camussi R., J. Fluid Mech., 383 (1999) 55.

[12] Kerr R. M. and Herring J. R., J. Fluid Mech., 419 (2000) 325.

[13] Xia K.-Q., Lam S., Zhou S.-Q. and Shang X.-D., oral communication at the Workshop on High Rayleigh Number Convection, Illmenau, 2001.

[14] Chavanne X., Chillà F., Castaing B., Hébral B., Chabaud B. and Chaussy J., Phys. Rev. Lett., 79 (1997) 3648.

[15] Niemela J. J., Skrbek L., Sreenivasan K. R. and Donnelly R. J., Nature, 404 (2000) 837.

[16] Wu X. Z., Along a Road to Developed Turbulence: Free Thermal Convection in Low Temperature Helium Gas, PhD Thesis, University of Chicago, 1991, unpublished.

[17] Roche P.-E., Castaing B., Chabaud B. and Hébral B., Phys. Rev. E, 63 (2001) 045303(R).

[18] Chavanne X., Chillà F., Chabaud B., Castaing B., Chaussy J. and Hébral B., J. Low Temp. Phys., 104 (1996) 109.

[19] This bridge was designed in our laboratory by Bret J. L. and FAure J. P., unpublished.

[20] McCarty R. D. and ArP V. D., in Advances in Cryogenic Engineering, edited by Fast R. W., Vol. 35 (Plenum Press, New York) 1990, pp. 1465-1475; Kierstead H. A., Phys. Rev. A, 3 (1971) 329; 7 (1973) 242; Moldover M. R., Phys. Rev., 182 (1969) 342; ACton A. and Kellner K., Physica B, 90 (1977) 192; 103 (1981) 212; Hands B. A. and ArP V. D., Cryogenics, 21 (1981) 697; Aziz R. A. et al., Phys. Rev. Lett., 74 (1995) 1586; Donnelly R. J. and BAREnghi C. F., University of Oregon report, Eugene, 1998; Steward W. G. and Wallace G. H., NIST report, Boulder, 1971; ARP V., private communication.

[21] Trelfall D. C., J. Fluid Mech., 67 (1975) 17.

[22] Chavanne X., Castaing B., Chillà F., Chabaud B. and Hébral B., Phys. Fluids, 13 (2001) 1300.

[23] Heslot F., Castaing B. and Libchaber A., Phys. Rev. A, 36 (1987) 5870.

[24] Cioni S., Ciliberto S. and Sommeria J., J. Fluid Mech., 335 (1997) 111. 